\title{
Walking in a Cube: Novel Metaphors for Safely Navigating Large Virtual Environments in Restricted Real Workspaces
}

\author{
Gabriel Cirio, Peter Vangorp, Emmanuelle Chapoulie, Maud Marchal, Anatole Lécuyer, and George Drettakis
}

\begin{abstract}
Immersive spaces such as 4-sided displays with stereo viewing and high-quality tracking provide a very engaging and realistic virtual experience. However, walking is inherently limited by the restricted physical space, both due to the screens (limited translation) and the missing back screen (limited rotation). In this paper, we propose three novel locomotion techniques that have three concurrent goals: keep the user safe from reaching the translational and rotational boundaries; increase the amount of real walking and finally, provide a more enjoyable and ecological interaction paradigm compared to traditional controller-based approaches. We notably introduce the "Virtual Companion", which uses a small bird to guide the user through VEs larger than the physical space. We evaluate the three new techniques through a user study with travel-to-target and path following tasks. The study provides insight into the relative strengths of each new technique for the three aforementioned goals. Specifically, if speed and accuracy are paramount, traditional controller interfaces augmented with our novel warning techniques may be more appropriate; if physical walking is more important, two of our paradigms (extended Magic Barrier Tape and Constrained Wand) should be preferred; last, fun and ecological criteria would favor the Virtual Companion.
\end{abstract}

Index Terms-Virtual reality, locomotion techniques, walking, restricted workspaces.

\section{INTRODUCTION}

Immersive spaces such as 4-sided (cube or CAVE-like [5]) displays with stereo viewing and high-quality tracking provide a very engaging and realistic virtual experience. Recent advances in tracking and display technologies have now made it possible to immerse users in complex and engaging Virtual Environments (VE), resulting in a very strong feeling of presence.

In many cases, walking inside immersive spaces improves presence [25], naturalness [25, 28] and task performance [28, 19]. However, such setups inherently limit real walking due to the restricted physical space. Users can only walk for a few steps before encountering the physical screens (limited translation), raising additional issues such as user safety, screen damage, tracking loss and visual artifacts. In addition, there is often no rear screen on the cube; when the activity in the VE requires the user to turn, their illusion of presence could be broken by the missing screens (limited rotation). Finally, locomotion is often achieved with a wand which is unnatural and permanently encumbers one hand.

Our goal is to address these limitations of cube-like immersive displays, namely 1) keep the user "safe" from reaching translational and rotational limits, 2) increase the amount of real walking for the aforementioned reasons, and 3) provide more enjoyable and ecological paradigms compared to traditional wand-based locomotion techniques. Ecological interfaces can be either real or virtual devices that fit into the concept of the virtual world and must behave in a natural and intuitive way.

At first glance, these goals may seem simple or just plain common sense. However, developing locomotion metaphors achieving these techniques can be surprisingly hard. There has been a significant body of work on walking in VEs, which we review in detail in Sect. 2. Most notably, for cube-like spaces, techniques such as "Walking in Place" [24] have been proposed; however they do not involve true physical walking. Several approaches have been developed for modifying the

- G. Cirio, M. Marchal and A. Lécuyer are with INRIA Rennes.

- P. Vangorp, E. Chapoulie and G. Drettakis are with REVES/INRIA Sophia-Antipolis

- M. Marchal is also with INSA Rennes.

- E-mail: first.last@inria.fr

Manuscript received 15 September 2011; accepted 3 January 2012; posted online 4 March 2012; mailed on 27 February 2012.

For information on obtaining reprints of this article, please send emailto:tvcg@computer.org.

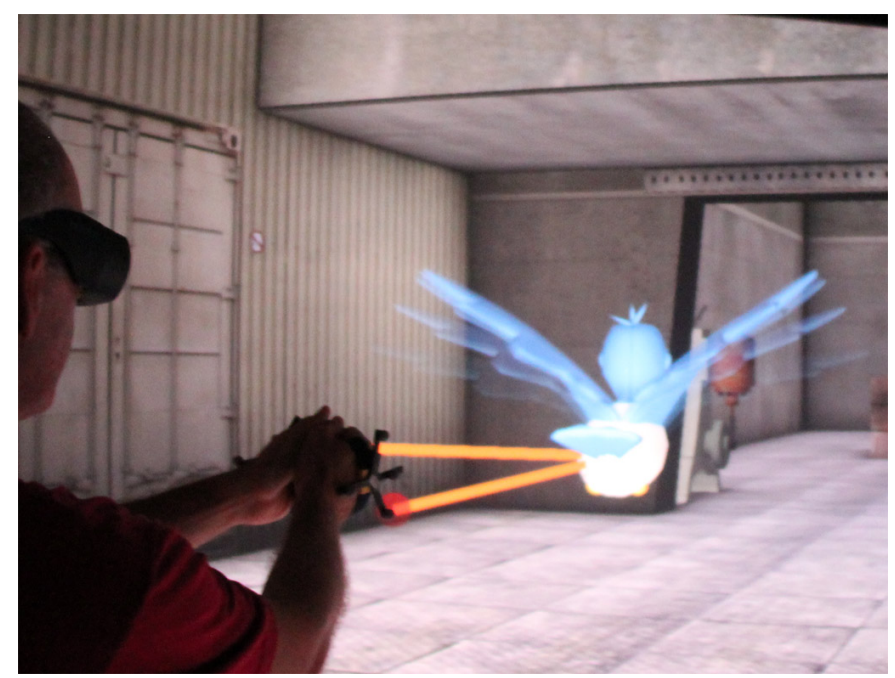

Fig. 1. One of the novel locomotion techniques we propose: the Virtual Companion is implemented with a bird which both warns the user about physical workspace limits and allows navigation in the environment using a metaphor with virtual reins.

walking path (e.g., "Redirected walking" [16]), but they usually require a space which is larger than a typical cube-like display. Among others, the "Magic Barrier Tape" [4] has been proposed to rid the user of the wand; however, it is not completely ecological, and does not directly apply to a cube.

In this paper, we propose three new techniques which address these limitations in different ways and to different degrees. First, they all incorporate a warning technique which prevents users from hitting the walls or seeing the missing screen. Second, they incorporate a $l o$ comotion technique which enables displacement to out-of-reach locations using a rate-control paradigm (similar to flystick or wand-based control). In two of the new techniques the rate-control is deactivated when the user is far from the walls, i.e., inside the "safety zone" of the cube, in order to maximize walking in this area. These three techniques were developed in a continuum, from less to more ecological: each technique is progressively more integrated with the virtual world.

The first metaphor, called the Constrained Wand and Signs extends the basic and well-known wand paradigm by adding virtual warning 
signs and forcing the user to walk to the limits of the space before activating the wand. The second metaphor extends the Magic Barrier Tape [4] to deal with the problem of the missing screen and the small physical space. Our third metaphor introduces a Virtual Companion which uses a bird to provide an even more ecological warning and locomotion metaphor (Fig. 1). We evaluate these three metaphors by comparing them first to a "base condition" which is a simple wand interface augmented with our novel virtual signs for safety reasons. We use two experimental methods to compare the techniques. First, we collect tracking data and analyze quantities such as walking distance, speed etc. Second, we use Likert-scale questionnaires to evaluate the impression of the user in terms of accuracy, walking sensation etc.

Our main contributions are thus: three novel metaphors for immersive navigation using restricted physical workspaces, namely 1) the Constrained Wand, 2) the Extended Magic Barrier Tape, and 3) the Virtual Companion; together with a comparative evaluation of these techniques at a "proof-of-concept" stage of usability, which provides insights into the relative strengths and weaknesses of each and should help developers choose the appropriate approach depending on their application needs. Most notably, our new metaphors introduce a solution to the problem of looking at the missing screen and present a novel locomotion interface paradigm in the form of the Virtual Companion.

The rest of the paper is organized as follows. After discussing previous work (Sect. 2), we give a detailed presentation of each technique (Sect. 3), followed by the description of our experiment and results (Sect. 4,5). We then discuss the results (Sect. 6) and conclude in Sect. 7.

\section{Previous work}

There is an important body of work on locomotion techniques for virtual reality $[1,2]$. We first review techniques which either allow locomotion with no physical motion of the user (static locomotion) or no spatial displacement (walking in place). We then discuss locomotion techniques involving natural walking, which are more relevant to our work.

\subsection{Static Locomotion and Walking in Place}

There are several known Virtual Reality (VR) locomotion metaphors where the user is not required to walk [2], and therefore do not need to overcome restricted workspace problems. Examples of these include teleportation i.e., an instantaneous change of position to a new location. Worlds In Miniature (WIM) [14] is a metaphor where the user has a copy of the virtual world in his hands. Users can choose a location in the copy and be smoothly taken there in the "real" virtual world. Probably the most common locomotion technique is the Flying Vehicle where the environment is not manipulated. The illusion is that the user can move through the world, either by using a mock-up, a wand or other device.

Walking in place $[20,24]$ simulates the physical act of walking without forward motion of the body; a virtual forward motion is introduced. Visual optical flow of navigation, that should be matched by proprioceptive information from the natural walking gait, is instead matched by proprioceptive information from a gait close to natural walking. The sense of presence is greatly increased compared to static locomotion techniques [25], but other sensory data related to walking, mainly vestibular cues, are still missing.

\subsection{Natural Walking}

Several studies have shown the benefits of using natural walking for the navigation of virtual environments, in terms of task performance $[19,8,28]$, presence [25] and naturalness [11, 25, 28]. Hence, several techniques try to adapt natural walking to restricted size workspaces.

In Step WIM [12] a user invokes a miniature version of the world at their feet, allowing distances greater than the physical environment to be traveled. The user can walk on the WIM to a new position and trigger a rescaling command that will scale up the WIM until it reaches the virtual world size. When used in a CAVE, where the field of view is restricted by the missing screen, a mapping of the $360^{\circ}$ of the scene to the system display field is used. The effect of the technique is noticed by the user, requiring a small adaptation time to adjust, and does not entirely solve the missing screen problem.

Resetting techniques $[26,27]$ try to overcome the limited working environment problem with natural walking using head-mounted displays (HMDs). They reset the user's position or orientation in the real world when reaching workspace limits, without breaking spatial awareness of the virtual world. In the freeze-backup technique, the virtual world is frozen and the user takes steps backwards to re-center her real world position inside the workspace. In the freeze-turn technique, the orientation of the user is frozen while she physically pivots $180^{\circ}$. In the $2: 1$-turn technique, a $360^{\circ}$ virtual rotation is mapped to a $180^{\circ}$ real world rotation, and the user also physically pivots $180^{\circ}$. These resetting techniques are performed consciously by the user following a warning signal, which implies a break in immersion. Moreover, the resetting itself might feel unnatural.

The Seven League Boots [9] allows natural walking in virtual worlds larger than the real space. It scales the user's speed only along their intended direction of travel, using gaze and previous displacement direction. Although well appreciated, it does not entirely solve the limited workspace problem.

The Magic Barrier Tape [4] explicitly displays the boundaries of the real environment within the virtual environment as virtual barrier tape. It allows natural walking locomotion within the space delimited by the tape, as well as rate control at the boundaries by pushing on the tape. It provides an easy, intuitive and safe way of navigating in a virtual scene, without breaking the sense of immersion, but does not solve the missing screen problem if used in a CAVE.

\subsubsection{Redirection techniques}

Redirected Walking [16, 21, 10] and Motion Compression [13, 7, 22] techniques "trick" the user into walking in a curved path in the real world when walking in a straight line in the virtual world through the progressive rotation of the scene around them. In a sufficiently large workspace, and with a straight virtual path, the user can walk endlessly without reaching the limits of the real workspace. These techniques are natural and in some cases imperceptible. However, they require large workspaces, can be confusing when doing unpredictable or quick changes of direction, and may require distracting events [15]. In practice, they are more suited for HMDs and wide area tracking systems.

Redirected Walking was used in combination with a walking in place technique in a CAVE environment to avoid looking at the missing screen [17]. Experiments showed that the frequency of looking at the missing screen was not reduced compared to hand-held navigation, although the variance was.

In the specific case of architectural virtual walkthroughs, the ArchExplore interface [3] allows the use of redirection techniques in small environments such as CAVEs. A virtual door mechanism is combined to increased (perceptible) redirection gains and the splitting of virtual rooms considered too large for redirection into smaller sized rooms. The missing screen problem is not addressed in this work.

In recent work, change blindness redirection [23] redirects users wearing HMDs by making dynamic changes to the environment, such as changing the configuration of doors and corridors while the user is focusing on a distracting task. The fact that the technique is not noticeable renders these results very promising.

There is clearly a lack of adequate locomotion techniques allowing immersive real walking and infinite exploration for CAVE-like environments. Existing techniques exhibit many limitations, and there is no convincing solution for the missing screen problem. Although very promising, redirection techniques require specific real and virtual environments in order to work correctly. In this work, we address these issues by proposing three novel locomotion techniques for safe, natural and enjoyable navigation within CAVE-like environments.

\section{Three novel Locomotion TeChNiques}

In the introduction, we presented three goals: 1) keep the user safe, 2) increase walking, 3) eliminate the wand and provide an ecological 

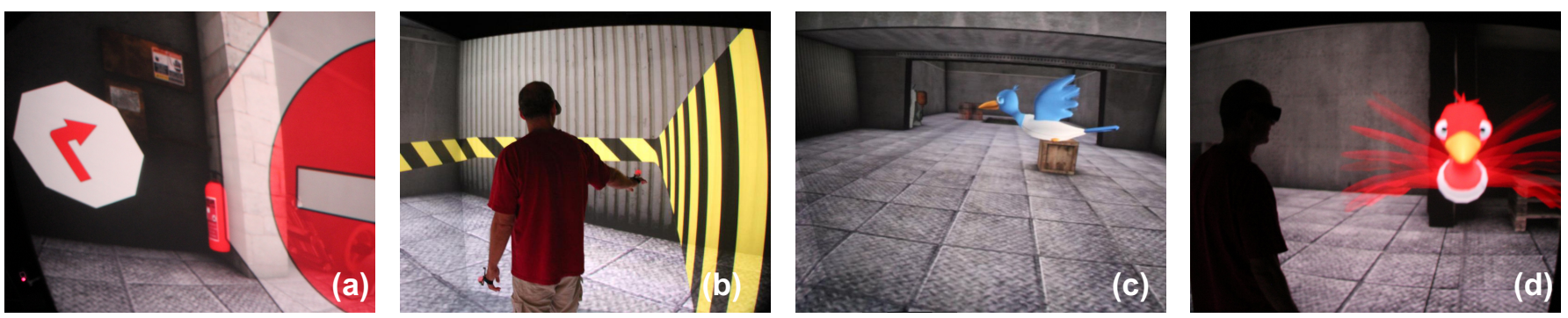

Fig. 2. Screenshots illustrating the three techniques. From left to right: (a) Constrained wand and signs: The "no-way" and "turn right" signs. (b) Extended MBT: the tape and blinders. (c,d) Virtual Companion: the bird in "rest mode" (c) and "protection mode" (d).

locomotion metaphor. In this section, we present our three new locomotion metaphors designed to achieve these goals. We encourage the reader to view the accompanying video for a better illustration of the metaphors.

\subsection{Motivation for New Locomotion Metaphors}

Our three novel metaphors can be seen as varying from simple (and trivial) to more complex, and from less to more ecological. The techniques are indeed progressively more embedded in the virtual environment. We wanted to avoid using rotation and translation scaling due to their noticeability in small evironments $[16,3]$ and their need of specific virtual environments. Instead, we used hybrid position/rate control approaches, based on the good results of previous locomotion techniques for limited workspaces [4].

The techniques we introduced were outlined in the introduction. For two of the techniques the rate-control mode is not possible when the user is located far from the boundaries of the workspace, i.e., inside the central (and thus safer) area. This satisfies our second goal by strongly encouraging the user to walk whenever they are located inside the safe area of the restricted workspace.

In the constrained wand (CWand) approach warning signs are presented before collisions (translation) or when turning the head too far (rotation). When the signs are visible the wand can be used as usual. This metaphor both encourages walking and keeps the user safe (Fig. 2(a), 4(left)).

In the Extended Magic Barrier (eMBT), the user can move beyond the physical space by pushing the barrier with their hand (Fig. 2(b)). By construction, the barrier only appears when approaching the walls or rotating too far. As a result this metaphor also encourages walking, keeps the user safe and removes the need for the wand.

The third novel metaphor is the Virtual Companion, which is an animated bird in the virtual environment. The bird serves two purposes: it protects the user at the limits by becoming red and flying close to the user's face (Fig. 2(d)), and can be "tethered" with virtual reins similar to the "Turtle Surf" technique [18], thus serving as a locomotion interface. This approach protects the user, leaves their hands free and is the most ecological of the three since the bird is part of the VE. However, it only slightly encourages walking, by forcing users to step backwards when they are too close to the screens or have turned too far.

\subsection{General Terminology and Quantities}

Before presenting the three techniques in detail, we introduce some terminology and the corresponding boundaries in the physical workspace. In our case, this is a 4-sided cube-shaped space (3 walls and the floor) measuring $3.2 \mathrm{~m}$ wide $\times 3.2 \mathrm{~m}$ long $\times 2.4 \mathrm{~m}$ high.

At a high level, we define a "safety zone" in which the user is not looking at the missing screen and is not close to the walls of the cube. We then define a "danger zone" in which there is a danger of physical collision with the walls or of seeing the missing screen, and a "reaction" zone in which boundary cues are progressively presented, warning the user that they are getting close to the danger zone. These zones are common to all techniques.

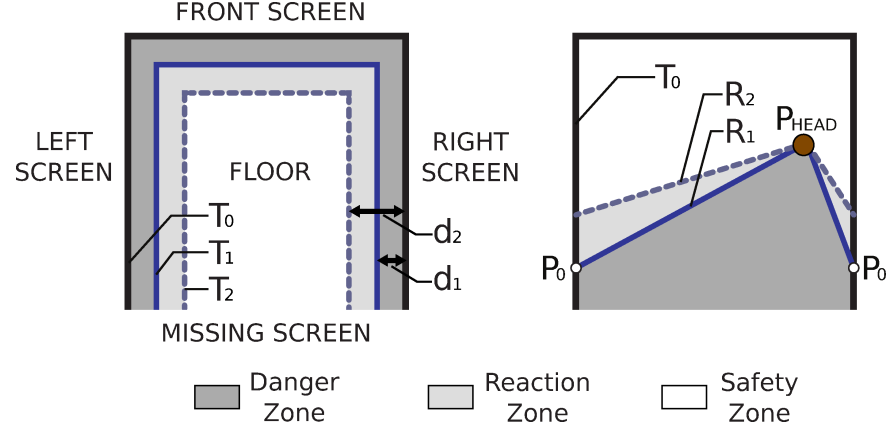

Fig. 3. Regions and boundaries for translation (left) and rotation (right) common to all techniques.

For translation, these zones are defined by the following translation boundaries, illustrated in Fig. 3(left).

- $T_{0}$ is the physical position of the walls.

- $T_{1}$ bounds the danger zone for translations, with $T_{1}=T_{0}-d_{1}$. The translation danger zone is between $T_{1}$ and $T_{0}$, where there is a risk of collision with the physical walls; we set $d_{1}=40 \mathrm{~cm}$ for our environment, empirically leaving enough space for comfortable arm motion.

- $T_{2}$ bounds the reaction zone, with $T_{2}=T_{0}-d_{2}$. The translation reaction zone is between $T_{2}$ and $T_{1}$, while the translation safety zone is between the workspace center and $T_{2}$. We set $d_{2}=75 \mathrm{~cm}$, which empirically allows ample warning for reasonably fast motion.

For rotation, these zones are defined as follows and illustrated in Fig. 3(right):

- Vector $R_{1}$ bounds the danger zone for rotations. It is defined by the vector $P_{0}-P_{\text {head }}$, where $P_{0}$ is a position on the side walls, and $P_{\text {head }}$ is the position of the user's head. $P_{0}$ was set at $60 \mathrm{~cm}$ from the missing screen after testing for an early warning and a correct rendering of the visual warning cues. When turning beyond $R_{1}$, the user is in the rotation danger zone. The angle between the head orientation and $R_{1}$ defines the angular distance from the user to the rotation danger zone.

- Vector $R_{2}$ bounds the reaction zone for rotations. It is defined by the angle $a_{0}$ to $R_{1}$. The rotation reaction zone is between $R_{2}$ and $R_{1}$, and the rotation safety zone is before $R_{2}$. We set $a_{0}=25^{\circ}$ considering a reasonably fast rotation motion of a user.

In rate control, the motion velocity goes from 0 to $1.5 \mathrm{~m} / \mathrm{s}$ in translation and from 0 to $1 \mathrm{rad} / \mathrm{s}$ in rotation. The control of the velocity is 
linear for all three techniques, but the input mechanism is different and adapted to each metaphor, as detailed in the locomotion paragraphs of the subsequent descriptions.

\subsection{Constrained Wands and Signs}

The constrained wand is illustrated in Fig. 4.

Warning A semi-transparent "no-way" sign appears when the user reaches $T_{2}$ and becomes fully visible at $T_{1}$. For rotation, a "turnleft" sign appears when the user turns right into the reaction zone (equivalently "turn-right"). Fig. 2(a) shows both visuals.

Locomotion Users can use the wand only in the reaction and danger zones. In these zones, the wand is operated in the customary manner. The forward translation direction is given by the wand direction.

Implementation For rotations, the sign position is in the viewing direction and in the reaction zone its distance varies linearly between $0.7 \mathrm{~m}$ and $1.2 \mathrm{~m}$ from the user's head position.
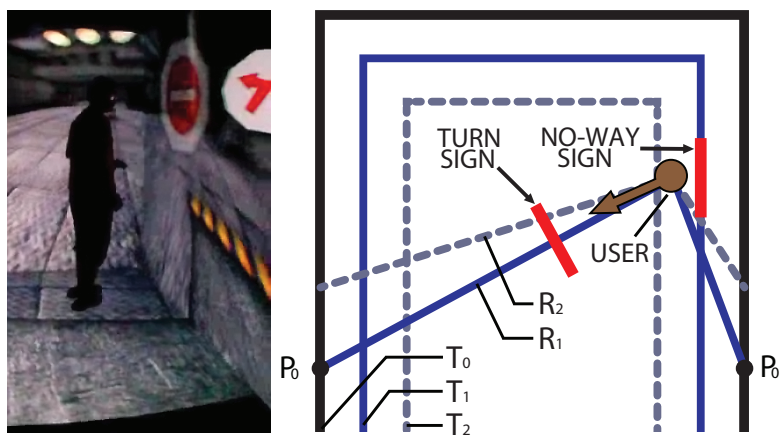

Fig. 4. The constrained wand: signs become visible to warn for rotation and translation.

\subsection{Extended Magic Barrier Tape}

The eMBT is illustrated in Fig. 5.

Warning The "translation tape" is located at $T_{1}$ and is aligned with the walls. It appears semi-transparently when the user is at $T_{2}$ and becomes fully visible at $T_{1}$. For rotation, we have introduced "virtual blinders". These start at the side of the translation tape (see Fig. 5(right)), and progressively become wider until they completely block the view of the user when reaching the head (see Fig. 2 (b)).

Locomotion The user translates by pushing the tape forward with a tracked hand. The speed depends linearly on how far the user pushes, and the direction is defined by the positions of the head and the hand. Rotation is achieved by a "pushing back" gesture on the blinders.

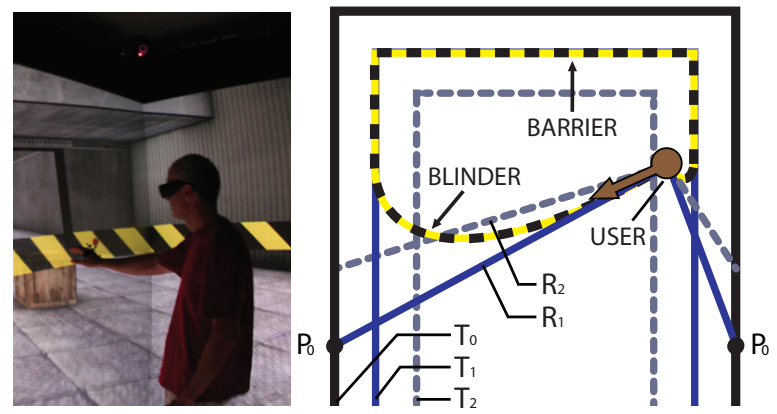

Fig. 5. Extended MBT: the tape follows the walls, and blinders warn for rotation.
Implementation We experimentally set the height ("altitude") of the tape to $1.4 \mathrm{~m}$; it is infinitely thin, has a width of $15 \mathrm{~cm}$ and a yellow/black slanted texture imitating real barrier tape. The blinders follow Bézier curves with the tape/blinder intersection, $P_{0}$ and the user head position as control points. The width of the blinder at the user's head is $60 \mathrm{~cm}$.

\subsection{Virtual Companion}

The Virtual Companion (VC) is the most innovative of the three techniques we introduce. An interactive "companion" is used to implement warning and locomotion (see Fig. 1, 2(c)-(d)). In our implementation we used a bird with flapping wings, but other representations could be used (dog, fairy etc.). In contrast to the previous techniques, locomotion can be achieved in the safety zone for the VC. However, if the user is in the danger zone, she must step back into the safety zone to initiate locomotion.

Warning When the user is in the safety zone, a blue bird stays near the closest wall, calmly flaps its wings and hovers at the height of the head (see Fig. 6, 2(c)). We call this "rest" mode. In the translation reaction zone, the bird's position is at the position of the user's head projected onto the closest wall. We call this "following mode". In the danger zone (translation and rotation), the bird flies directly in front of the user's face, turns red and angrily flaps its wings (Fig. 2(d) and video). This is "protection mode".
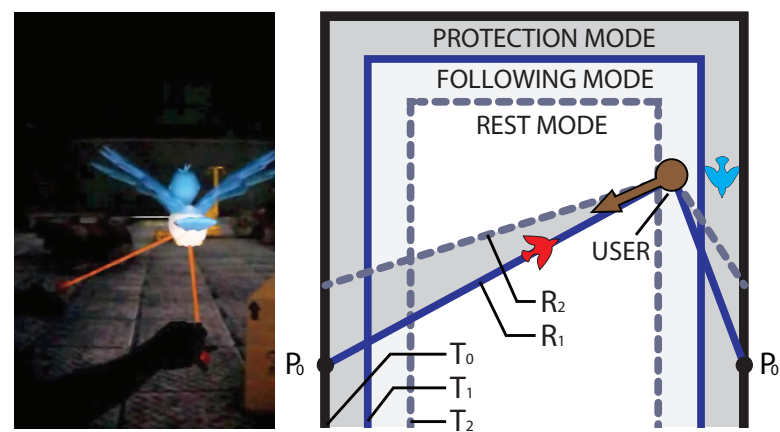

Fig. 6. The Virtual Companion. Left: the gesture for turning. Right: the bird changes position and color to warn for rotation and translation.

Locomotion We introduce a set of gestures to navigate with the VC using virtual reins. These are illustrated in Fig. 7. The user invokes "navigation mode" by bringing their hands together for 1 second. The reins then appear, attaching the user's hands to the VC. The VC moves in front of the user. To cancel navigation mode, the user crosses her arms. To move forward the user imitates a move forward command that would be given to a horse (moving the reins up and down). Once the motion has started, the user moves their hands forward to accelerate, and pulls back to decelerate and eventually stop. Rotation is achieved by moving the corresponding hand to the side. The rotation speed is given by the distance between the hands. To stop turning, the active hand is moved back next to the other hand. The gestures can be seen in the accompanying video. The implemented gestures are given as examples but future work could focus on the best way to control the Virtual Companion.

Implementation The bird follows Bézier curves using the current position and past and future goal positions for smooth interpolation between trajectories. Rotation protection has priority over the translation protection, except when reaching $T_{1}$ where priorities are inverted. The move forward gesture is the most sensitive: the user has $1 \mathrm{~s}$ to complete an up-down movement of $25 \mathrm{~cm}$ with both hands.

\section{Evaluation}

To evaluate the different metaphors described above, we compare the three new locomotion techniques, namely Constrained Wand, Ex- 


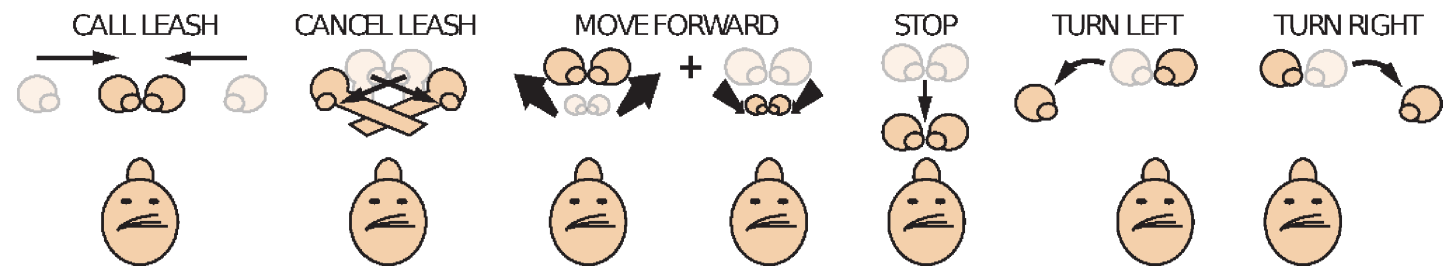

Fig. 7. The gesture set for controlling the Virtual Companion.

tended Magic Barrier Tape, and Virtual Companion. We also include in the comparison the "baseline condition" of a standard Wand interface, typically used for navigation in CAVE-like workspaces, which is expected to be faster and possibly more accurate. The only enhancement for the wand is the appearance of warning signs for collision, thus avoiding physically bumping into walls. We chose this approach to provide a fair comparison; in typical workspaces the experimenter/operator uses a verbal warning to avoid collisions which adds a modality unavailable in the other techniques.

We conducted two experiments corresponding to two different tasks, a travel-to-target task and a path following task. Both tasks were performed first in a simple scene with minimal visual cues to distance and orientation (Fig. 8), and later in a complex scene with more realistic visual cues (Fig. 1). The aim of the travel-to-target task was to evaluate the walking distances during the navigation in the virtual environment. We expected that the Extended Magic Barrier Tape and Constrained Wand will encourage users to walk more than the other techniques. The aim of the path following task was to test the locomotion in all directions, including the missing screen. We expected that the Virtual Companion will help users to stay in the safety zone.

Population Twelve participants (3 females and 9 males) aged from 23 to $61(M=30.7, S D=10.0)$ took part in this experiment. Three of them were left-handed. Three of them wore glasses or contact lenses to correct for myopia. None had any other known vision or perception disorders. They were all unpaid volunteers and naïve to the purpose of the experiment.

Experimental Apparatus The experiment was conducted in a 4screen cube ( 3 walls and the floor) measuring $3.2 \mathrm{~m}$ wide $\times 3.2 \mathrm{~m}$ long $\times 2.4 \mathrm{~m}$ high. Each screen uses Infitec stereo running at $60 \mathrm{~Hz}$ per eye, and was driven by a separate NVIDIA Quadro 5800 GPU to ensure a constant $60 \mathrm{fps}$ per eye. The position and orientation of the user's head and hands and of the wand were tracked by an ART 6-camera infrared tracking system running at $60 \mathrm{~Hz}$.
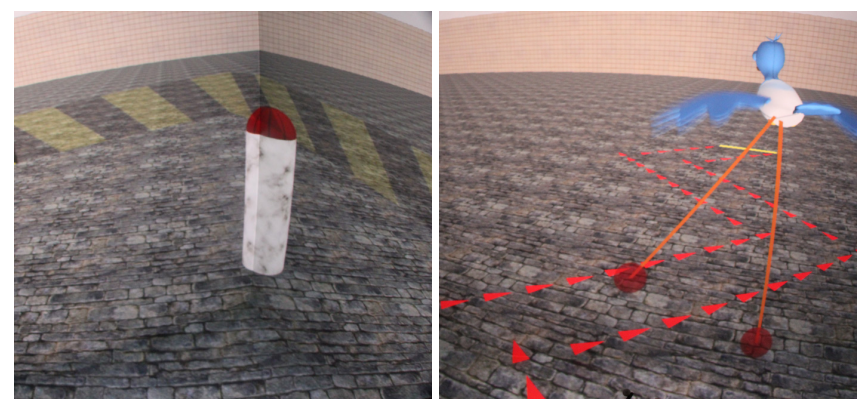

Fig. 8. The simple environment used for the first block of tests in our study. Left: simple target object, right: zigzag path.

Two scenes of different complexity were constructed for the purpose of these experiments. The simple scene consisted of a large square room $(50 \mathrm{~m} \times 50 \mathrm{~m})$ with a stone floor texture, tiled walls, and an untextured ceiling (see Fig. 8). The complex scene was an industrial hangar with a central open area where the targets were placed (Fig. 1,2). Outside the experiment area, it was cluttered with machines, crates, and barrels, and two more rooms were visible. This scene had realistic distances and sizes, and high resolution textures with baked global illumination.

For travel-to-target tasks, a target was placed in the scene. In both scenes, the target was a marble cylinder with a red hemispherical cap resembling a button on top. For path following tasks, the edges of the path were marked on the floor by red arrows indicating the direction to follow, and a yellow finish line indicated the end of the path. These can be seen in Fig. 8 and in the video.

Procedure Before the beginning of the experiment, the four techniques were explained to the subject in a training session taking approximately $15 \mathrm{~min}$ on average. The training scene consisted of the simple scene, in which a $3 \mathrm{~m}$ path with a single $90^{\circ}$ corner was marked on the floor and three targets successively appeared. This training session accustomed participants to the workspace, the display technology, the locomotion techniques, and the travel-to-target and path following tasks.

In the experiment, participants first performed a block of travel-totarget and path following tasks in the simple scene, and then a second block in the complex scene. Between the two blocks, there was a pause to fill in a questionnaire of 11 Likert items. This pause also served to avoid cybersickness from prolonged use of the workspace. After both blocks were completed, a second, subjective questionnaire of open questions concluded the experiment. Each block of trials lasted about $15 \mathrm{~min}$, and the complete experiment, including training and questionnaires, lasted approximately $60 \mathrm{~min}$ on average.

Collected data For each trial and each subject, we recorded the completion time (in seconds) and all the head tracker readings (i.e., position and orientation of the head). This data also allows the computation of the amplitude of physical walking (in meters), and the time spent in the danger zone. The amplitude of physical walking is the distance traveled when walking in the real world, which of course also causes an equal traveled distance in the virtual world. It also allows the computation of the path deviation from the ideal path, i.e., the shortest path to the target or the center line of the indicated path. The path deviation $\left(\mathrm{in}^{2}\right.$ ) is given by the area delimited by the subject's path in the virtual scene and the ideal path.

\subsection{Task \#1: Travel to Target Task}

In Task \#1, our goal was to compare the 4 techniques ( 3 new and Wand) in a travel-to-target task where the user had to move from a central initial location to a new location, indicated by a target, as fast as possible. When the target is reached, the user touches the target with one hand and the task is completed.

Procedure Before each trial, the subject had to go back to the initial position and orientation of the real workspace (the middle position between the side screens, at $40 \mathrm{~cm}$ from the missing screen and facing the front screen). Then, the scene became visible and the participant was instructed to look for the target in the scene, move towards it, and touch it with one hand. The participant was instructed to use physical walking or virtual walking using the technique, or a combination of both, at their own choice. The target was always placed outside the limits of the physical workspace, so some amount of virtual walking was always required to complete the task. 


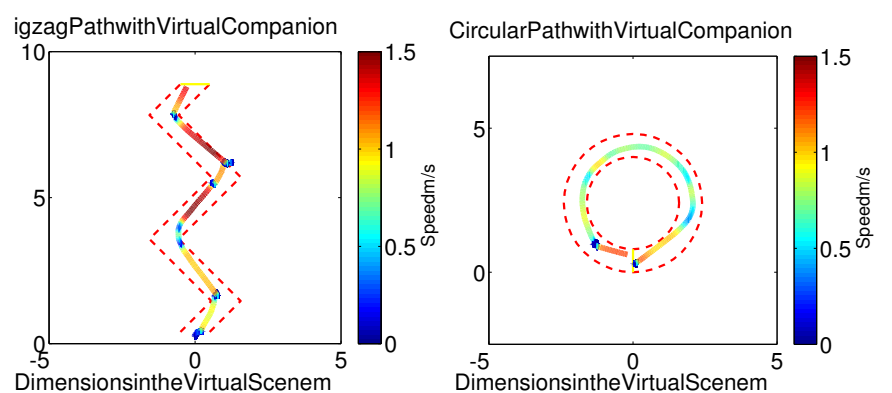

Fig. 9. Recorded trajectories of a participant performing (a) a zigzag path and (b) a circular path. The trajectories are the result of physical walking and/or Virtual Companion navigation and are color coded with the speed.

Participants completed all four conditions (corresponding to the four locomotion techniques) and the order of the conditions was counterbalanced across participants. In each condition, the participants were exposed to 2 trials, with the target either at $3.6 \mathrm{~m}$ straight in front of the user's initial position, or $4.4 \mathrm{~m}$ straight behind, alternating throughout the experiment. Participants completed a total of 8 trials (2 target positions $\times 4$ technique conditions) in each of the 2 scenes.

\subsection{Task \#2: Path Following Task}

In Task \#2, our goal was to compare the 4 techniques in a path following task where the user had to follow a path delimited by red arrows on the virtual ground (see Fig. 8(right)), as fast as possible and as accurately as possible by trying to stay in the middle of the path.

Procedure Before each trial, the subject had to go back to the initial position and orientation. Then, the scene became visible and the participant was instructed to follow the path in the direction indicated by the arrows on the floor, until they reached the finish line. Walking instructions were the same as the first task, and again some virtual walking was necessary since the path extended beyond the physical space.

As for Task \#1, participants completed all conditions in counterbalanced order. In each condition, the participants were exposed to 2 trials, either with a zigzag path $12 \mathrm{~m}$ long and $70 \mathrm{~cm}$ wide, with four $90^{\circ}$ corners (Fig. 9(a)), or with a circular path $12.5 \mathrm{~m}$ long and $80 \mathrm{~cm}$ wide (Fig. 9(b)), alternating throughout the experiment. Participants completed a total of 8 trials ( 2 paths $\times 4$ technique conditions) in each of the 2 scenes.

\section{REsULts}

We first present the results from time and tracking data recorded during the experiments and then the results of the subjective questionnaire: these are results only for the simple scene. We summarize the differences between the simple and the hangar scenes at the end of this section.

\subsection{Recorded time and tracking data}

Considering the experimental protocol, Friedman tests were used to test for differences among the 4 navigation techniques according to several criteria. Post-hoc comparisons were performed using Wilcoxon signed-rank tests with a threshold of 0.05 for significance. Reported p-values are adjusted for multiple comparisons. The results are also visualized compactly in Fig. 10.

Time to complete the task The time needed to complete the tasks differed significantly across the 4 techniques for all the tasks: target task $\left(\chi^{2}=2.85, p=0.02\right)$, zigzag path $\left(\chi^{2}=3.64, p=0.002\right)$ and circular path $\left(\chi^{2}=4.43, p<0.001\right)$. For the target task, post-hoc analysis revealed that Wand was significantly faster than VC $(p=0.02)$ Separating target in front and behind, there is no significant effect for behind target, while for front target post-hoc analyis gives Wand faster

\begin{tabular}{|c|c|c|c|}
\hline $\begin{array}{c}\text { VC: } 76.7 \mathrm{~s} \\
(\mathrm{SD}=38.4 \mathrm{~s})\end{array}$ & $\begin{array}{l}\text { eMBT: } 68.2 \mathrm{~s} \\
(\mathrm{SD}=44.1 \mathrm{~s})\end{array}$ & $\begin{array}{c}\text { CWand: } 34.3 \mathrm{~s} \\
(\mathrm{SD}=12.6 \mathrm{~s})\end{array}$ & $\begin{array}{c}\text { Wand: } 22.4 \mathrm{~s} \\
(\mathrm{SD}=10.3 \mathrm{~s})\end{array}$ \\
\hline
\end{tabular}

(a) Completion time, zigzag path

\begin{tabular}{|c|c|c|c|}
\hline $\begin{array}{l}\text { eMBT: } 60.2 \mathrm{~s} \\
(\mathrm{SD}=22.2 \mathrm{~s})\end{array}$ & $\begin{array}{c}\text { VC: } 43.4 \mathrm{~s} \\
(\mathrm{SD}=22.4 \mathrm{~s})\end{array}$ & $\begin{array}{l}\text { CWand: } 36.5 \mathrm{~s} \\
(\mathrm{SD}=24.9 \mathrm{~s})\end{array}$ & $\begin{array}{c}\text { Wand: } 21.0 \mathrm{~s} \\
(\mathrm{SD}=6.3 \mathrm{~s})\end{array}$ \\
\hline
\end{tabular}

(b) Completion time, circle path

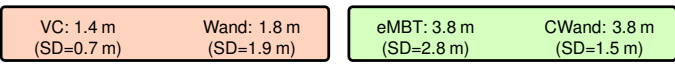

(c) Physical walking distance, both target tasks

$\left.\begin{array}{|cc|}\hline \begin{array}{c}\text { VC: } 1.5 \mathrm{~m} \\
(\mathrm{SD}=0.7 \mathrm{~m})\end{array} & \begin{array}{c}\text { Wand: } 2.4 \mathrm{~m} \\
(\mathrm{SD}=2.5 \mathrm{~m})\end{array}\end{array}\right]$\begin{tabular}{cc|}
$\begin{array}{cc}\text { CWand: } 4.2 \mathrm{~m} & \text { eMBT: } 4.8 \mathrm{~m} \\
(\mathrm{SD}=1.8 \mathrm{~m}) & (\mathrm{SD}=3.6 \mathrm{~m})\end{array}$ \\
\hline
\end{tabular}

(d) Physical walking distance, target behind

\begin{tabular}{|cccc|}
$\begin{array}{c}\text { Wand: } 3.9 \mathrm{~m} \\
(\mathrm{SD}=1.9 \mathrm{~m})\end{array}$ & $\begin{array}{c}\text { CWand: } 6.5 \mathrm{~m} \\
\text { VC: } 6.8 \mathrm{~m}\end{array}$ & $\begin{array}{c}\text { eMBT: } 11.3 \mathrm{~m} \\
(\mathrm{SD}=3.2 \mathrm{~m})\end{array}$ & $\begin{array}{c}\text { (SD }=4.5 \mathrm{~m}) \\
(\mathrm{SD}=4.5 \mathrm{~m})\end{array}$ \\
\hline
\end{tabular}

(e) Physical walking distance, zigzag path

\begin{tabular}{|c|c|c|c|}
\hline $\begin{array}{l}\text { VC: } 3.2 \mathrm{~m} \\
(\mathrm{SD}=2.7 \mathrm{~m})\end{array}$ & $\begin{array}{l}\text { Wand: } 3.6 \mathrm{~m} \\
(\mathrm{SD}=2.8 \mathrm{~m})\end{array}$ & $\begin{array}{c}\text { CWand: } 7.4 \mathrm{~m} \\
(\mathrm{SD}=4.1 \mathrm{~m})\end{array}$ & $\begin{array}{c}\text { eMBT: } 10.0 \mathrm{~m} \\
(\mathrm{SD}=3.4 \mathrm{~m})\end{array}$ \\
\hline
\end{tabular}

(f) Physical walking distance, circle path

\begin{tabular}{|cccc|}
\hline $\begin{array}{c}\text { CWand: } 37.3 \% \\
(\mathrm{SD}=10.8 \%)\end{array}$ & $\begin{array}{c}\text { eMBT: } 27.8 \% \\
(\mathrm{SD}=17.7 \%)\end{array}$ & $\begin{array}{l}\text { Wand: } 9.5 \% \\
(\mathrm{SD}=11.5 \%)\end{array}$ & $\begin{array}{c}\text { VC: } 9.2 \% \\
(\mathrm{SD}=9.1 \%)\end{array}$ \\
\hline
\end{tabular}

(g) Time spent in warning mode, target in front

Fig. 10. Visualization of the results: boxes are ordered by the results of post-hoc tests. Separate boxes indicate statistically significant differences. We also provide the means and standard deviations for each corresponding condition for clarity. (a)-(b) Time needed to complete the zigzag path and the circular path. (c)-(f) Physical walking distance for the 4 tasks. (g) Amount of time spent in reaction and danger zones during the travel to target task with the target in front.

than CWand. For the path following task, post-hoc analysis for the zigzag path revealed that Wand was significantly faster than eMBT $(p=0.009)$ and VC $(p=0.001)$. For the circle path, Wand was only significantly faster than eMBT $(p<0.001)$. The speed advantage of Wand was possibly because of the precision required for locomotion.

Physical walking distance The amplitude of physical walking differed significantly across the 4 techniques for each task: $\operatorname{target}\left(\chi^{2}=\right.$ $3.79, p<0.001)$, zigzag path $\left(\chi^{2}=4.27, p<0.001\right)$, and circular path $\left(\chi^{2}=3.79, p<0.001\right)$. For the target tasks, post-hoc analysis showed that eMBT encourages physical walking more than $\mathrm{VC}(p<0.001)$ and Wand $(p=0.008)$. Participants also walked significantly more with CWand compared to VC $(p<0.001)$ and Wand $(p=0.008)$.

There are significant effects for the separate target tasks. For the front target, CWand incites people to walk more than VC $(p=0.01)$ and eMBT incites people to walk more than VC $(p=0.003)$ and Wand ( $p=0.01)$. For the target behind, CWand encourages walking more than VC $(p=0.01)$, and eMBT encourages walking more than VC $(p=0.003)$ and Wand $(p=0.01)$. For the path following task, posthoc analysis revealed that for the zigzag path eMBT encourages more physical walking than Wand $(p<0.001)$. For the circle path, post-hoc analysis showed that eMBT encourages physical walking more than VC $(p<0.001)$ and Wand $(p=0.001)$. However, we observed that in some cases the user walked to the danger zone once and just stayed there because there was no incentive to return to the safe zone. The VC and especially the Wand do not incite users to walk physically at all. Recorded trajectories of where the participants moved in the physical workspace are included in the supplemental material.

Time spent in reaction and danger zones The amount of time spent in the zones where the warning visual cues are active, both in rotation and translation, differed significantly across the 4 techniques 
for each task: target $\left(\chi^{2}=4.43, p<0.001\right)$, zigzag path $\left(\chi^{2}=4.43\right.$, $p<0.001)$ and circular path $\left(\chi^{2}=5.06, p<0.001\right)$. For the target tasks, post-hoc analysis revealed that the time spent in reaction and danger zones was significantly lower for VC compared to CWand $(p<0.001)$ and eMBT $(p=0.04)$ and for Wand compared to CWand $(p<0.001)$. There is also a significant effect for the separate target tasks. For target in front, users spent less time in these zones with VC than with CWand $(p=0.003)$ and with Wand than CWand $(p<0.001)$. For target behind, eMBT and VC reduced their time in reaction and danger zones compared to CWand ( $p=0.04$ and $p<0.001$ respectively). For the zigzag path following task, post-hoc analysis showed that the time spent in reaction and danger zones was significantly lower for VC compared to CWand $(p<0.001)$ and eMBT $(p=0.03)$ and for Wand compared to CWand $(p=0.002)$. For the circular path following task, post-hoc analysis revealed that the participants spent less time in the reaction and danger zones for VC compared to CWand $(p<0.001)$ and eMBT $(p=0.08)$, eMBT compared to CWand $(p=0.04)$ and Wand compared to CWand $(p=0.01)$.

Closer examination of the actions that trigger the warning cues reveals that the translation warning seems to be dominant. The CWand and the eMBT force users to enter the reaction zone closer to the limits of the physical workspace to activate the technique. Users almost never look in the direction of the missing screen while using the eMBT. Also, users almost never approach the limits of the physical workspace when using the VC, and its statistic is actually dominated by the rotation reaction and danger zones.

Deviation from the ideal path The deviation from the ideal path was measured by calculating the area between the ideal and actual paths. This metric mainly penalizes users for deviating from the ideal path over longer stretches, such as cutting corners. The deviation from the ideal path did not differ significantly across the 4 techniques for both types of task

Scene differences The same experimental protocol was conducted in a Hangar scene. Overall the effects observed in the simple scene are confirmed for the Hangar scene. In particular we obtain the same significant effect for time to complete the task. For time in danger/reaction zones, only the walk-to-target task differs: we do not have a significant effect for eMBT for the complex scene. For the physical distance, we do not have a significant effect for the complex scene when the target is behind in what concerns Wand vs. eMBT. On the other hand we do have a new significant effect for the path following task, i.e., for the zigzag path, eMBT encourages more physical walking than VC $(p<0.001)$ and Cwand more than Wand $(p=0.02)$. For the circle path, CWand encourages more physical walking than VC $(p=0.008)$ and Wand $(p=0.04)$. Given these observations, and since participants find visually rich scenes more enjoyable, we encourage the use of such scenes for this type of locomotion interface studies.

\subsection{Questionnaire}

After completing both tasks in the simple scene only, a preference questionnaire was proposed in which participants had to grade the four techniques from 1 (low) to 7 (high) according to 11 subjective criteria: (a) Accuracy, (b) Ease of use, (c) Fun, (d) Walking sensation, (e) Fatigue, (f) Speed, (g) Presence, (h) Cybersickness, (i) Safety (avoid bumping into walls), (j) Missing screen awareness, (k) Global appreciation. The questionnaire is included as supplemental material. Figure 11 shows the results concerning the grades (Likert-scale) obtained by the four different techniques for 4 subjective criteria. By performing a Friedman test on the four different conditions, we found a significant effect for 8 criteria, including Accuracy $\left(\chi^{2}=9.89, p=0.019\right)$, Ease of use $\left(\chi^{2}=16.54, p<0.001\right)$, Fun $\left(\chi^{2}=19.36, p<0.001\right)$, Walking sensation $\left(\chi^{2}=16.34, p<0.001\right)$, and Missing screen awareness $\left(\chi^{2}=9.23, p=0.03\right)$. No significant effect was found for Cybersickness $(\chi 2=0.40, p=0.94)$; however, participants reported very low levels of cybersickness $(M=1.73, S D=1.41)$.

Post-hoc analysis showed that the Wand was rated significantly higher than the eMBT for accuracy $(p=0.009)$ and ease of use $(p=0.003)$. The Wand was also rated significantly higher than the VC
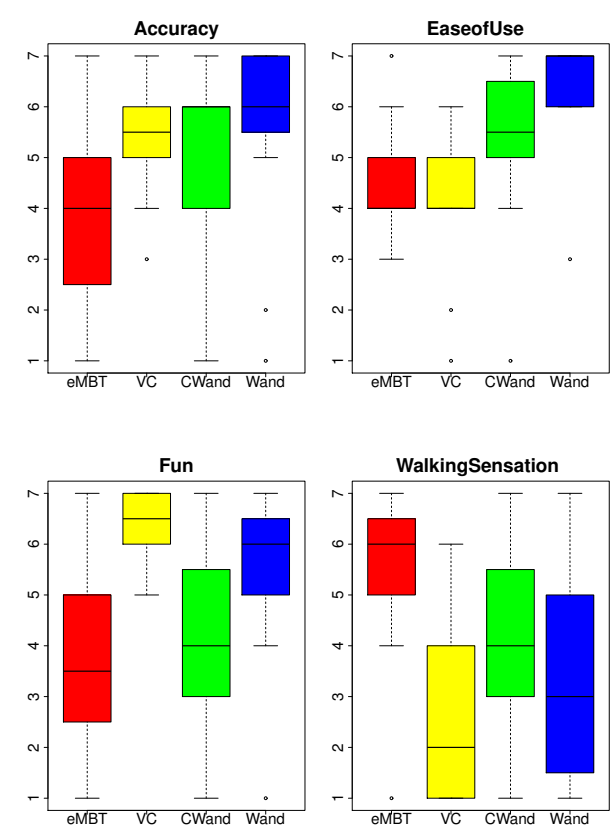

Fig. 11. Boxplots comparing the grades of the four techniques for some of the most interesting subjective criteria in the questionnaire.

for ease of use ( $p=0.003)$. The eMBT was rated significantly higher than the Wand for missing screen awareness $(p=0.03)$ and walking sensation $(p=0.01)$. The eMBT was also rated significantly higher than the VC for walking sensation $(p=0.001)$. Finally, the VC was rated significantly higher than the CWand for fun $(p=0.007)$. The VC was also rated significantly higher than the eMBT for fun $(p<0.001)$.

\section{Discussion}

We proposed three novel interaction techniques for locomotion in CAVE-like environment: an improved version of the Wand technique constraining the use of the wand to unsafe zones, an extended version of the Magic Barrier Tape [4] adapted to CAVE-like environments and a completely novel metaphor called Virtual Companion to assist the user during his navigation. Our goals were: (1) to keep the user safe, (2) to encourage the user to walk and (3) to provide ecological interaction techniques.

Concerning the first goal, participants never hit the wall with any of the four techniques. We believe this was due to good user training and to the effectiveness of our methods. When comparing the techniques among them, we see that the Virtual Companion outperforms the eMBT and the CWand in terms of staying in the "safety zone" (Fig. 10(g)). Inevitably, this comes at the price of lower physical walking distance for all tasks except the zigzag path (Fig. 10(c)(e)). However, in this case the additional walking was mainly due to position adjustments during navigation. Although participants spent more time in the protection zone for the eMBT compared to the VC, they gave higher ratings in the questionnaire to the criterion "missing screen awareness" compared to the Wand. Participants found the eMBT metaphor useful for warning them of the real workspace limits, as previously observed in the original version [4].

For the second goal, the eMBT induces the largest amount of physical walking for three out of four tasks, compared to VC and Wand. This is also confirmed by the subjective questionnaire, where the technique was ranked higher than both the Wand and the $\mathrm{VC}$ in terms of walking sensation. In addition, both eMBT and CWand outperform the $\mathrm{VC}$ and the Wand in terms of physical walking distance when considering both target tasks (Fig. 10(c)). For the eMBT, this is also confirmed when taking the tasks separately, except for the zigzag path 
where it outperforms only the Wand. The eMBT thus meets the initial goal of proposing new metaphors allowing users to walk in restricted real workspaces.

For the third goal, evaluating the success of the ecological nature of the new metaphors is harder. The VC was judged to be significantly more fun than the CWand and the eMBT; interestingly there was no significant difference with the Wand. It must be noted however that the VC scored low on ease of use; learning the gestures did require some effort, and, like any gesture-based interface, tracking quality is paramount. In some cases tracking did deteriorate, causing some subject frustration especially for the start gesture. Nonetheless, both the eMBT and the VC succeed at providing a controller-free locomotion metaphor, hence improving user interaction and freeing the hands for other tasks. Interestingly, subjects sometimes used the VC "rein" control while walking; this shows that the VC is very well integrated in the virtual environment, underlining its ecological nature.

The presence question did not provide any significant results. However, in the free-form comments, 8 out of 12 subjects included positive remarks ("fun", "great" etc.) about the VC, while no such comments were given on the other techniques. The VC was also rated significantly higher than the eMBT and CWand for the "fun" criterion. We interpret this as an encouraging indication on approaches such as the VC. The "fun" aspect of the companion as the assistance tool provided to the participants instead of warning signs might represent a good alternative to existing techniques.

Nonetheless and as expected, the Wand technique tends to be overall faster and more accurate, both in terms of objective measures (completion time, Fig. 10(a)-(b)), and from the subjective responses in the questionnaire (Sect. 5.2).

It is interesting to note that the goals of providing safety and encouraging walking can be contradictory: when using a wand, it is possible to go everywhere without moving or turning. Thus the safety goal is completely achieved but walking is not encouraged. We believe that this observation indicates a deeper issue which is confirmed by our results.

A second issue which follows from our results is that more complex -and in our case more ecological-interfaces come at the price of slower speed in task accomplishment. This was true both for eMBT and VC. To a lesser extent there is also an effect on task accuracy.

Both these issues are tradeoffs which needs to be considered by application designers. Table 1 provides an overview of the different techniques we have proposed, summarizing the relative strengths and weaknesses of each. It could help developers choose the appropriate approach depending on their application needs. The different criteria reflect the main aspects that were evaluated and discussed in this paper, grouped by goal. This overview takes into account statistical data as well as subjective feedback and observations made during the experiment. As such it should be considered cautiously. Still, it seems that if speed and accuracy are paramount, more traditional controller interfaces may still be appropriate, but augmented with our novel signs and warning techniques for improved safety. If physical walking is more important, using a paradigm such as eMBT or CWand should be preferred. Last, ecological criteria (fun, no wand, integration in the environment) would favor the $\mathrm{VC}$ and its gestural interaction with a friendly virtual character.

\section{Conclusions}

We have presented a study of locomotion in restricted physical immersive workspaces. We set out to achieve three goals, namely keeping the user safe, encouraging walking and providing ecological navigation.

We presented three new techniques, the Constrained Wand, the Extended Magic Barrier Tape and the Virtual Companion. We designed and ran a user study to compare the relative advantages of each approach, and compare to a traditional wand "baseline" condition. Overall our three new techniques all achieve at least two of the initial goals. They all achieve safety in translation to some extent, since we did not observe collision issues. The CWand and the eMBT also achieve increased walking. The $\mathrm{VC}$ achieves ecological interface to some extent,

\begin{tabular}{|c|c|c|c|c|c|c|}
\hline Goal & Criter & & Wand & CWand & eMBT & VC \\
\hline 1 & Safety & $\begin{array}{l}\text { translation } \\
\text { rotation }\end{array}$ & $\begin{array}{c}++ \\
+\end{array}$ & $\begin{array}{l}+ \\
+\end{array}$ & $\begin{array}{c}+ \\
++\end{array}$ & $\begin{array}{c}++ \\
+\end{array}$ \\
\hline 2 & \multicolumn{2}{|c|}{ Physical walking } & -- & + & ++ & -- \\
\hline 3 & $\begin{array}{l}\text { Requir } \\
\text { Fun } \\
\text { Presen }\end{array}$ & wand & $\begin{array}{l}- \\
+ \\
?\end{array}$ & $\begin{array}{l}- \\
- \\
?\end{array}$ & $\begin{array}{c}++ \\
- \\
?\end{array}$ & $\begin{array}{c}++ \\
++ \\
?\end{array}$ \\
\hline Other & $\begin{array}{l}\text { Fast na } \\
\text { Ease o } \\
\text { Learni }\end{array}$ & $\begin{array}{l}\text { igation } \\
\text { use } \\
\text { time }\end{array}$ & $\begin{array}{l}++ \\
++ \\
++\end{array}$ & $\begin{array}{c}+ \\
+ \\
++\end{array}$ & $\begin{array}{l}- \\
- \\
+\end{array}$ & $\begin{array}{l}- \\
- \\
-\end{array}$ \\
\hline
\end{tabular}

Table 1. Summary of main advantages/drawbacks of our techniques.

since it does not require a wand and subjective grades for "fun" and user free-from comments highlighted its engaging nature.

We have notably introduced a solution to the problem of seeing the missing screen in cube-like immersive spaces. This is confirmed by our study which indicates in particular that when using the eMBT method users almost never look the missing screen.

We believe that our study provides interesting insight into the various tradeoffs involved in locomotion techniques for restricted immersive spaces. We have introduced three new metaphors, each having different comparative strengths and weaknesses. We are particularly hopeful about the Virtual Companion paradigm and its potential for future navigation methods.

Of course, an ideal locomotion interface for restricted spaces should be able to achieve all three goals simultaneously. One possible direction for this is to have a more reactive "intelligent" virtual companion; however such a research direction adds numerous challenges. The Virtual Companion technique could also be tested using other representations. For instance, more "human" creatures, such as a fairy or an angel, could represent a virtual "guardian angel". These creatures could justify the use of additional and promising verbal feedback. Additional sensory feedback could be incorporated, such as auditory effects (known to increase warning performances [29]) or haptic feedback (known to increase performance of rate-control [6]). The gestures controlling the VC could be improved, taking into account ergonomics and usability criteria.

Future work could also focus on further evaluations of our techniques to inform about their usability in concrete VR applications. Different fields could be tested (industry, medicine, architecture, etc.) with context-based metaphors matching the requirements and representations used in every particular application field.

\section{ACKNOWLEDGMENTS}

This research was supported in part by the INRIA ARC NIEVE, and the European Community under FP7 FET-Open grant agreement $\mathrm{n}^{\circ} 222107$ NIW - Natural Interactive Walking. Additional support was generously provided from Adobe, Autodesk and NVIDIA. E. Chapoulie was partly supported by the Provence Alpes Côte d'Azur Region. The authors wish to thank all the participants in the experiments.

\section{REFERENCES}

[1] L. L. Arns and C. Cruz-Neira. A new taxonomy for locomotion in virtual environments. In Virtual Reality International Conference, pages 141149, 2002.

[2] D. A. Bowman, E. Kruijff, J. J. LaViola, and I. Poupyrev. 3D User Interfaces: Theory and Practice. Addison-Wesley Professional, 1 edition, 2004.

[3] G. Bruder, F. Steinicke, and K. H. Hinrichs. Arch-Explore: a natural user interface for immersive architectural walkthroughs. In IEEE Symposium on 3D User Interfaces, 2009. 3DUI 2009, pages 75-82. IEEE, 2009.

[4] G. Cirio, M. Marchal, T. Regia-Corte, and A. Lécuyer. The magic barrier tape: a novel metaphor for infinite navigation in virtual worlds with 
a restricted walking workspace. In Proceedings of the 16th ACM Symposium on Virtual Reality Software and Technology, pages 155-162, Kyoto, Japan, 2009. ACM.

[5] C. Cruz-Neira, D. J. Sandin, T. A. DeFanti, R. V. Kenyon, and J. C. Hart. The CAVE: audio visual experience automatic virtual environment. Communications of the ACM, 35:64-72, 1992.

[6] L. Dominjon, A. Lécuyer, J. Burkhardt, and S. Richir. A comparison of three techniques to interact in large virtual environments using haptic devices with limited workspace. Advances in Computer Graphics, 4035:288-299, 2006.

[7] D. Engel, C. Curio, L. Tcheang, B. Mohler, and H. H. Bülthoff. A psychophysically calibrated controller for navigating through large environments in a limited free-walking space. In Proceedings of the 2008 ACM symposium on Virtual reality software and technology, pages 157-164, Bordeaux, France, 2008. ACM.

[8] M. Heintz. Real walking in virtual learning environments: Beyond the advantage of naturalness. In U. Cress, V. Dimitrova, and M. Specht, editors, Learning in the Synergy of Multiple Disciplines, volume 5794, pages 584-595. Springer Berlin Heidelberg, Berlin, Heidelberg, 2009.

[9] V. Interrante, B. Ries, and L. Anderson. Seven league boots: A new metaphor for augmented locomotion through moderately large scale immersive virtual environments. In Proceedings of the IEEE Symposium on 3D User Interfaces, pages 167-170, 2007.

[10] L. Kohli, E. Burns, D. Miller, and H. Fuchs. Combining passive haptics with redirected walking. In Proceedings of the 2005 international conference on Augmented tele-existence, pages 253-254, Christchurch, New Zealand, 2005. ACM.

[11] W. L. Kuan and C. Y. San. Constructivist physics learning in an immersive, multi-user hot air balloon simulation program (iHABS). In $A C M$ SIGGRAPH 2003 Educators Program, page 1. ACM Press, 2003.

[12] J. J. LaViola, D. A. Feliz, D. F. Keefe, and R. C. Zeleznik. Hands-free multi-scale navigation in virtual environments. In Proceedings of the ACM symposium on Interactive 3D graphics, pages 9-15. ACM, 2001.

[13] N. Nitzsche, U. D. Hanebeck, and G. Schmidt. Motion compression for telepresent walking in large target environments. Presence: Teleoper. Virtual Environ., 13(1):44-60, 2004.

[14] R. Pausch, T. Burnette, D. Brockway, and M. E. Weiblen. Navigation and locomotion in virtual worlds via flight into hand-held miniatures. In Proceedings of ACM SIGGRAPH, pages 399-400. ACM, 1995.

[15] T. Peck, M. Whitton, and H. Fuchs. Evaluation of reorientation techniques for walking in large virtual environments. In Virtual Reality Conference, 2008. VR '08. IEEE, pages 121-127, 2008.

[16] S. Razzaque, Z. Kohn, and M. C. Whitton. Redirected walking. In Proceedings of Eurographics, 2001.

[17] S. Razzaque, D. Swapp, M. Slater, M. C. Whitton, and A. Steed. Redirected walking in place. In Proceedings of the workshop on Virtual environments 2002, pages 123-130, Barcelona, Spain, 2002. Eurographics Association.

[18] H. Reckter, C. Geiger, J. Singer, and S. Streuber. Tech-note: Iterative design and test of a multimodal experience. In IEEE Symposium on $3 D$ User Interfaces, 2009. 3DUI 2009, pages 99-102. IEEE, 2009.

[19] R. A. Ruddle and S. Lessels. The benefits of using a walking interface to navigate virtual environments. ACM Trans. Comput.-Hum. Interact., 16(1):1-18, 2009.

[20] M. Slater, M. Usoh, and A. Steed. Taking steps: the influence of a walking technique on presence in virtual reality. ACM Trans. Comput.-Hum. Interact., 2(3):201-219, 1995.

[21] F. Steinicke, G. Bruder, T. Ropinski, and K. H. Hinrichs. Moving towards generally applicable redirected walking. In Proceedings of the Virtual Reality International Conference (VRIC), pages 15-24. IEEE Press, 2008.

[22] J. Su. Motion compression for telepresence locomotion. Presence: Teleoper. Virtual Environ., 16(4):385-398, 2007.

[23] E. A. Suma, D. M. Krum, and M. Bolas. Redirection on mixed reality walking surfaces. In IEEE VR Workshop on Perceptual Illusions in Virtual Environments, page 33-35, 2011.

[24] J. N. Templeman, P. S. Denbrook, and L. E. Sibert. Virtual locomotion: Walking in place through virtual environments. Presence: Teleoper. Virtual Environ., 8(6):598-617, 1999.

[25] M. Usoh, K. Arthur, M. C. Whitton, R. Bastos, A. Steed, M. Slater, and J. Frederick P. Brooks. Walking $>$ walking-in-place $>$ flying, in virtual environments. In Proceedings of the 26th annual conference on Computer graphics and interactive techniques, pages 359-364. ACM Press/Addison-Wesley Publishing Co., 1999.
[26] B. Williams, G. Narasimham, B. Rump, T. P. McNamara, T. H. Carr, J. Rieser, and B. Bodenheimer. Exploring large virtual environments with an HMD when physical space is limited. In Proceedings of the ACM symposium on Applied perception in graphics and visualization, pages 41-48, Tubingen, Germany, 2007. ACM.

[27] X. Xie, Q. Lin, H. Wu, G. Narasimham, T. P. McNamara, J. Rieser, and B. Bodenheimer. A system for exploring large virtual environments that combines scaled translational gain and interventions. page 65. ACM Press, 2010.

[28] C. A. Zanbaka, B. C. Lok, S. V. Babu, A. C. Ulinski, and L. F. Hodges. Comparison of path visualizations and cognitive measures relative to travel technique in a virtual environment. IEEE Transactions on Visualization and Computer Graphics, 11(6):694-705, 2005.

[29] Y. Zhang, T. Fernando, H. Xiao, and A. R. L. Travis. Evaluation of auditory and visual feedback on task performance in a virtual assembly environment. Presence: Teleoperators and Virtual Environments, 15:613626, 2006 . 